\title{
Screening cellular metabolic activity
}

\section{To the Editor:}

A paper published by Gregory

Stephanopoulos and colleagues ${ }^{1}$ in your May 2014 issue described a microfluidic device for screening metabolic activity of cells. The authors put special emphasis on the fact that they had developed a fully integrated microfluidic platform allowing the encapsulation of cells into droplets that can be incubated, injected into a second device, fused and sorted.

However, this approach was not introduced by the authors, but had originally been published by us ${ }^{2}$ in 2012. In our paper, we even concluded that our approach "should facilitate metabolic studies." The general concept of combining different droplet manipulation modules into a single, fully integrated, cell screening device was patented even earlier ${ }^{3}$, with a priority date of

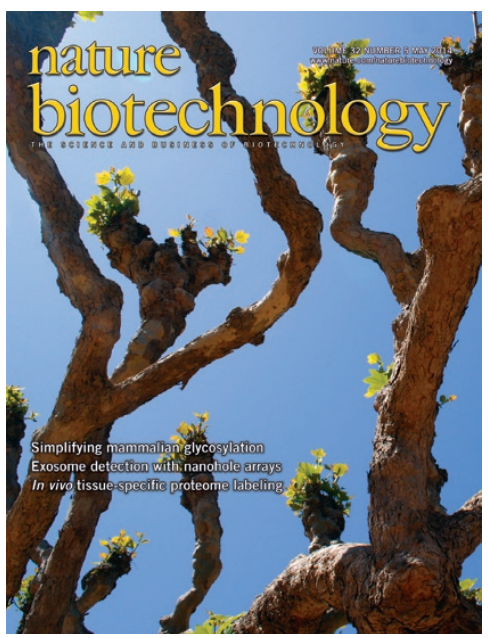

production or consumption. In this scenario, the culture and assay steps cannot be performed simultaneously because the time scale for production or consumption is much longer than the assay time, which is on the order of seconds or minutes. Thus, a separate step is necessary to culture each individual cell in its own droplet."

In our approach, the droplets did not contain assay reagents, but rather cultivation media. Exactly as Stephanopoulos and colleagues described in the Nature Biotechnology article $^{1}$, we added the assay reagents later, subsequent to a cultivation step during which extracellular proteins were produced.

"Here, we have described a system to measure extracellular metabolite secretion or consumption, which uses a microfluidic droplet maker to encapsulate cells and

July 13, 2007. However, neither our paper nor the patent was cited by Stephanopoulos and colleagues.

We also contend that many statements made by Stephanopoulos and colleagues are wrong. For example, they state, "Although others have demonstrated the use of microfluidic emulsion droplet technology to screen populations of cells, these previous applications have been limited to screening cells that have already completed their culturing process and that produce an analyte of interest physically connected to the cell by being either intracellular or membrane-bound." In our approach ${ }^{2}$, hybridoma cells were screened for the functional activity of secreted, soluble antibodies. The culturing process was not completed at the time of encapsulation, and the analyte of interest was neither intracellular nor membrane-bound, but a secreted one, thus contradicting the aforementioned claims.

In addition, the authors ${ }^{1}$ go on to state, "In these examples, the initial formation of droplets involved the addition of only assay reagents to determine the activity of the enzyme. This is very different from the detection of extracellular metabolite growth medium, a syringe for multi-day microaerobic culturing of collected droplets, and a second microfluidic device containing coalescence, delay line, detection and sorting modules. In this second device, the reagents to detect the analyte of interest are added to the droplets collected in the syringe; the assay reaction occurs while the droplets are in the delay lines; and droplets are sorted and collected based on resulting fluorescence." This is exactly the approach we first described in the paper by El Debs et al. ${ }^{2}$. It is noteworthy that we also obtained a fivefold higher throughput and an approximately twofold better enrichment than Stephanopoulos and colleagues did in their work ${ }^{1}$.

Our conclusion is that many aspects of the system described in their paper are not novel, and the authors should have cited all relevant previous work.

COMPETING FINANCIAL INTERESTS

The authors declare no competing financial interests.

Bachir el Debs, Ramesh Utharala \& Christoph A Merten

European Molecular Biology Laboratory (EMBL), Genome Biology Unit, Meyerhofstrasse 1, 69115

Heidelberg, Germany.

e-mail:christoph.merten@embl.de
1. Wang, B.L. et al. Nat. Biotechnol. 32, 473-478 (2014).

2. El Debs, B., Utharala, R., Balyasnikova, I.V. Griffiths, A.D. \& Merten, C.A. Proc. Natl. Acad. Sci. USA 109, 11570-11575 (2012)

3. Weitz, D.A. et al. Droplet-based selection. US patent 20090068170 (2009)

\section{Gregory Stephanopoulos and colleagues} reply:

El Debs and Merten ${ }^{1}$ contend that "this approach" (i.e., of an integrated microfluidic device described in our paper ${ }^{2}$ allowing the encapsulation of cells into droplets, which can be incubated, injected into a second device, fused and sorted) was not introduced by us. Furthermore, they claim that "the general concept of combining different droplet manipulation modules into a single, fully integrated, cell screening device was patented even earlier, with a priority date of July 13, 2007.” We rebut their claims on priority with the following timeline of events.

First, we initially publicly presented the results of our complete microfluidic system in 2008 using xylose-consuming yeast cells in a talk at the American Chemical Society (ACS) 236th National Meeting on August 19, 2008 (ref. 3). This presentation was followed by subsequent presentations at Metabolic Engineering VII (ME-VII) on September 17, 2008, and the 2008 American Institute of Chemical Engineers (AIChE) Annual Meeting on November 17, 2008 (refs. 4,5).

Second, regarding our failure to cite their patent ${ }^{6}$, we note that their own paper ${ }^{7}$ also failed to cite the patent, even though two of the co-authors are also listed as coinventors on the patent. More to the point, El Debs and Merten cite the priority date of the patent, which is irrelevant as this patent did not publish and therefore was not part of the public record, until March 12, 2009. In the meantime, we also filed patent application US20100124759 with priority date June 27, 2008, reporting xylose and glucose enrichment results with a complete integrated microfluidic droplet cell screening system ${ }^{8}$. It is of note that the Weitz patent ${ }^{6}$ above only mentions the concept of a complete integrated microfluidic system. No data with an integrated sorting module were provided.

Third, complete xylose screening results with details of the microfluidic droplet system were reported in Benjamin Wang's thesis on May 26, 2009, which has been 